\title{
APLICAÇÃO DO MÉTODO MULTICRITÉRIO SAPEVO-M NA SELEÇÃO DE EQUIPAMENTOS: ESTUDO DE CASO EM UMA PANIFICADORA DO RJ
}

\author{
Marcos dos Santos \\ Instituto Militar de Engenharia (IME) \\ Praça Gen. Tibúrcio, 80 - Urca, Rio de Janeiro - RJ, 22290-270 \\ marcosdossantos_doutorado_uff@yahoo.com.br \\ Nathália Campos de Oliveira \\ Universidade do Grande Rio (UNIGRANRIO) \\ Rua Professor José de Souza Herdy, 1160, Duque de Caxias - RJ, 25071-202 \\ nathaliacampos3@unigranrio.br \\ Pamela Ferreira Carvalho de Oliveira \\ Universidade do Grande Rio (UNIGRANRIO) \\ Rua Professor José de Souza Herdy, 1160, Duque de Caxias - RJ, 25071-202 \\ pamelaolivera@unigranrio.br \\ Carlos Francisco Simões Gomes \\ Universidade Federal Fluminense (UFF) \\ Rua Passos da Pátria, 156, Bloco D, São Domingos, Niterói, RJ. CEP: 24210-240 \\ cfsg1@bol.com.br
}

\section{RESUMO}

Atualmente o setor de panificação se encontra entre os seis maiores segmentos da indústria brasileira e o segundo maior em alimentos prontos. As transformações econômicas, as mudanças no hábito de consumo e a evolução dos mercados concorrentes pressionaram a indústria, com isso trazendo grandes desafios para o setor. O objetivo deste estudo é de ranquear um conjunto de alternativas de freezers disponíveis no mercado que atendam aos critérios pré-estabelecidos da padaria Doce Alto. A metodologia utilizada foi o SAPEVO-M, que é um método multicritério de apoio à tomada de decisão capaz de assessorar o agente decisor em diversos problemas complexos, avaliando, ordenando ou selecionando alternativas mediante diferentes pontos de vista. Os cálculos do método SAPEVO-M foram realizados com o auxílio do site www.sapevoweb.com. Neste trabalho o método auxiliou na seleção entre três modelos de freezers através de diferentes tomadores de decisão que avaliaram alguns critérios. Os resultados contribuirão para a seleção do melhor modelo de freezer que irá compor o processo produtivo da fabricação dos pães. Dessa forma, é possível dizer que essa ferramenta possui uma gama de aplicações, sendo capaz de contribuir no processo de tomada de decisão em diferentes seguimentos.

Palavra-chave: Apoio Multicritério à Decisão (AMD); Seleção de Freezeres; Padaria; Método SAPEVO-M; Aplicativo Computacional SAPEVO Web. 


\begin{abstract}
Currently the bakery sector is among the six largest segments of Brazilian industry and the second largest in ready foods. Economic transformations, changes in consumer habits and the evolution of competing markets have put pressure on the industry, thus posing major challenges for the industry. The aim of this study is to rank a set of commercially available freezer alternatives that meet the pre-established criteria of Doce Alto bakery. The methodology used was SAPEVO-M, which is a multicriteria decision support method capable of assisting the decision maker in several complex problems, evaluating, ordering or selecting alternatives through different points of view. The SAPEVO-M method calculations were performed with the help of www.sapevoweb.com. In this work the method assisted in the selection among three freezer models through different decision makers who evaluated some criteria. The results will contribute to the selection of the best freezer model that will compose the production process of bread making. Thus, it is possible to say that this tool has a range of applications, being able to contribute to the decision-making process in different segments.
\end{abstract}

Keywords: Multicriteria Decision Aid (MCDA); Selection of Freezers; Bakery; SAPEVOM Method; SAPEVO Web Computing Application.

\title{
Como Citar:
}

SANTOS, Marcos dos; OLIVEIRA, N. C.; OLIVEIRA, P. F. C.; GOMES, C. F. S. Aplicação do Método Multicritério SAPEVO-M na seleção de equipamentos: estudo de caso em uma panificadora do RJ. In: SIMPÓSIO DE PESQUISA OPERACIONAL E LOGÍSTICA DA MARINHA, 19., 2019, Rio de Janeiro, RJ. Anais [...]. Rio de Janeiro: Centro de Análises de Sistemas Navais, 2019.

\section{INTRODUÇÃO}

O cenário contemporâneo para as empresas de panificação e confeitaria brasileiras se mostra cada vez mais competitivo. Deste modo, enfrentar episódios de conflitos internos não é desejável. Em consequência da contínua entrada de padarias inovadoras no mercado, da colocação de novos profissionais mais capacitados tecnicamente e do aumento do grau de exigência dos consumidores, a competitividade no setor de panificação e confeitaria tem aumentado consideravelmente. Isso faz com que muitos profissionais do ramo busquem aperfeiçoamento na esfera gerencial do seu negócio para acompanhar a evolução e as exigências do mercado.

Sabe-se que com o crescimento nos últimos anos das indústrias de congelados permitiu-se uma ampliação do número de pontos de venda de produtos panificados. Com isso, os consumidores não compram pão somente nas padarias, existe uma migração para outros canais de venda, como supermercados, lojas de conveniência, mercadinhos, entre outros comércios.

Com o intuito de se adaptar à evolução do mercado e as várias exigências do consumidor moderno, as padarias devem oferecer uma gama de produtos. Portanto, a qualidade de processos, produtos e serviços tem sido considerada elemento fundamental para a competitividade empresarial. As organizações devem concentrar esforços para atender as necessidades e superar as expectativas consideradas importantes pelos clientes e garantir a conformidade com especificações em suas operações (TEIXEIRA et al., 2014). 
Vale salientar, entretanto, que empresas de panificação de menor porte apresentam desafios e questões peculiares que dificultam a aplicação das propostas convencionais constantes da literatura sobre gestão, que, em geral, são desenvolvidas para a realidade das grandes empresas, incluindo aquelas relacionadas à padronização de processos (TEIXEIRA et al., 2014).

O estudo e a proposição de práticas de gestão, principalmente aquelas voltadas para a gestão da qualidade e padronização, para pequenas empresas, característica da grande maioria das panificadoras brasileiras, devem ser realizados considerando efetivamente suas especificidades. Elas, de uma forma geral, são mais centralizadas, possuem estrutura organizacional simples, têm menor controle e defesa sobre ações provenientes do ambiente externo e tendem a personalizar a gestão na pessoa de seu proprietário-dirigente, que normalmente centraliza o poder de decisão e prefere ser guiado pela sua experiência e intuição ao invés de utilizar o ferramental teórico-prático disponível na literatura sobre administração (TEIXEIRA et al., 2014)

Em conformidade com o relatório de tendências de 2019 da Associação Brasileira da Indústria de Panificação e Confeitaria - ABIP observou-se que modernizar as áreas de produção, venda e os aspectos gerenciais das padarias estão atrelados à sustentabilidade das empresas. Porém do balcão para dentro, pensar em inovação significa também atender o cliente em seus variados momentos de aquisição. As padarias brasileiras são mundialmente conhecidas pela ampla oferta de produtos panificados, itens de revenda - empório - e ainda por oferecer serviços que acompanham as demandas dos clientes ao longo do dia. Inovação é também acompanhar as tendências do mercado no que se refere às vendas.

Paralelamente, vale ressaltar que aquelas padarias e confeitarias que procuram ao longo dos anos se atualizar, criando maior profundidade na oferta de produtos, mantendo alto padrão de qualidade e diversificação de serviços sentem menos o impacto e a concorrência trazida por esses novos competidores.

\section{DESCRIÇÃO DO PROBLEMA}

A panificadora e confeitaria Doce Alto localizada no Estado do Rio de Janeiro é um estabelecimento de pequeno porte atendendo aos consumidores locais e às pequenas empresas do entorno.

Sabe-se que a produção do pão envolve técnica apurada para o desenvolvimento de melhores produtos com características peculiares. Dessa forma, para se destacar no mercado é preciso oferecer produtos saborosos e de melhor qualidade.

O processo de fabricação dos pães está atrelado às diversas etapas que vão desde a elaboração até ao aperfeiçoamento das receitas. O mesmo ocorre através das escolhas de bons ingredientes e tipos de fermentação até o tempo de forno necessário para completar o processo final.

Nesse sentido, é fundamental para o sucesso do estabelecimento estar atento às questões internas com o intuito de prestar o melhor serviço e consequentemente à satisfação do cliente. Diante disso, é imprescindível que as panificadoras tenham sempre todos os equipamentos em perfeito estado de conservação e funcionamento.

Existem diversas etapas para produzir os pães e uma delas é a passagem pelo freezer, vale ressaltar que é nessa fase que surge o processo crítico. Posto que, é através deste equipamento que ocorre o retardamento da fermentação fazendo com que sempre haja pães frescos disponíveis para o consumo dos clientes. 
A padaria apresentada possui algumas dificuldades internas que estão relacionadas à fabricação dos pães e uma delas é a problemática referente ao freezer. No estabelecimento existem três freezers que compõem a estrutura de equipamentos utilizados para realizar a produção dos pães. Observou-se que dois desses instrumentos possuem capacidade de produção satisfatória para a demanda diária, ou seja, estão em perfeito estado de funcionamento. Já o terceiro, encontra-se inoperante, pois o mesmo apresenta problemas mecânicos.

Paralelamente, com essa ocorrência nota-se que a demanda sofreu diversas variações. Antes, quando havia o funcionamento dos três equipamentos era possível atender a demanda local e à das empresas do entorno. Com essa alteração no quadro de maquinários não é mais possível obter o mesmo resultado. A grande preocupação com relação à queda da demanda reflete na imagem do estabelecimento no mercado. Nesse sentido, tudo precisa estar de acordo com as expectativas dos clientes, uma vez que o menor erro ou um pequeno descontentamento é o suficiente para que os eles troquem a padaria pelo concorrente.

De acordo com o mapa mental apresentado na Figura 1 verifica-se as condições de contorno do problema. O mesmo tem por objetivo organizar e analisar o conteúdo de forma mais compreensível.

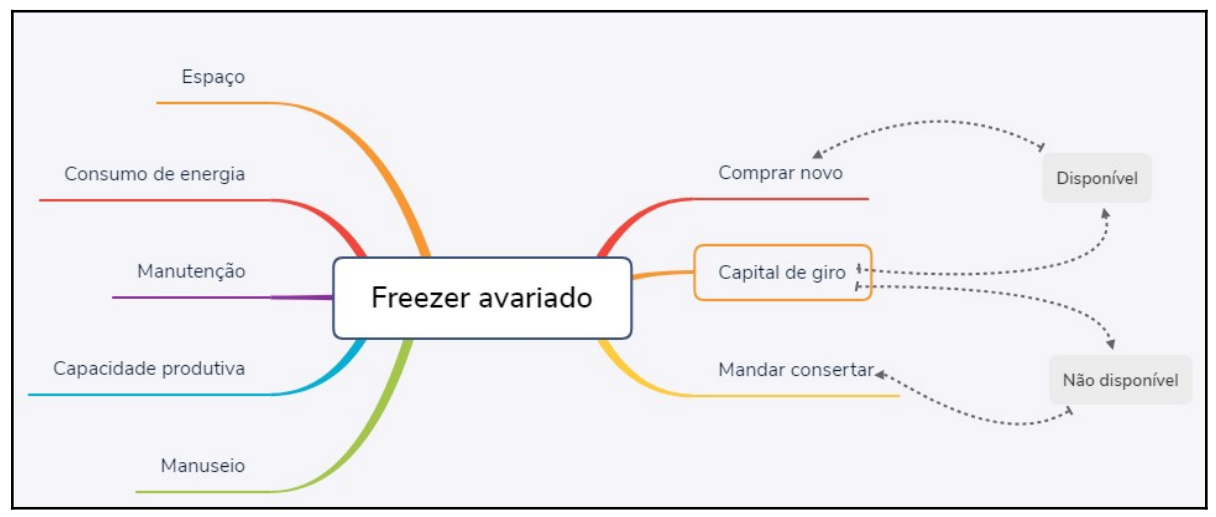

Figura 1: Mapa mental com as condições de contorno do problema. Fonte: Autores (2019).

Dado o exposto, esta pesquisa visa responder a seguinte questão: como ranquear um conjunto de alternativas de freezers disponíveis no mercado que atendam aos critérios preestabelecidos pela empresa?

\section{FUNDAMENTAÇÃO TEÓRICA}

\subsection{PANIFICADORAS}

A história mostra que o pão é conhecido do ser humano há, pelo menos, sete mil anos antes de cristo. O pão foi introduzido no Brasil anos mais tarde, trazido pelos europeus, no século XIX, mas foi somente no século XX, que o ramo da panificação expandiu no país.

Segundo Azevedo (2003) a França assumiu no Século XVII a liderança na indústria da panificação, seguida pela Áustria. No Brasil, o estado de Minas Gerais foi o pioneiro na indústria da panificação, principalmente devido à forte influência da imigração italiana. Seu início ocorreu já no final do século XIX e se expandiu rapidamente para os demais estados da confederação (SEBRAE, 2008). 
De acordo com Ferreira, Amorim e Maciel (2004), o mercado de panificação e confeitaria atual é dinâmico. Diante disso, é importante estar sempre atento às diversas variáveis que podem influenciar de certa forma os resultados deste ramo de atividade. A competitividade global existente na atualidade do mercado exige estratégias capazes de fazer com que as empresas alcancem melhor posicionamento dentro de seus ramos de atuação.

Esse ambiente demanda informações relevantes e de cunho estratégico relacionando custos, desempenho, processos, produtos, serviços e clientes (SANTOS, 2007).

\subsection{PESQUISA BIBLIOMÉTRICA}

A pesquisa bibliométrica foi realizada por meio do portal de periódicos da CAPES (Coordenação de Aperfeiçoamento de Pessoal de Nível Superior)/Scopus que é a maior base de dados de resumos e citações de literatura revisada por pares, com ferramentas bibliométricas para analisar, acompanhar e visualizar a pesquisa (Periódico CAPES/Scopus, 2016), tendo como objetivo demonstrar a importância que o tema abordado possui. Para construção dos gráficos, a pesquisa foi delimitada no período de 1990 a 2018 e foram empregadas as palavras multicritério e tomada de decisão.

De acordo com o Gráfico 1, pode-se observar que foi realizada uma pesquisa de documentos relacionados ao tema abordado comparando 10 países. O Brasil está na terceira colocação com 70 publicações referentes ao assunto.

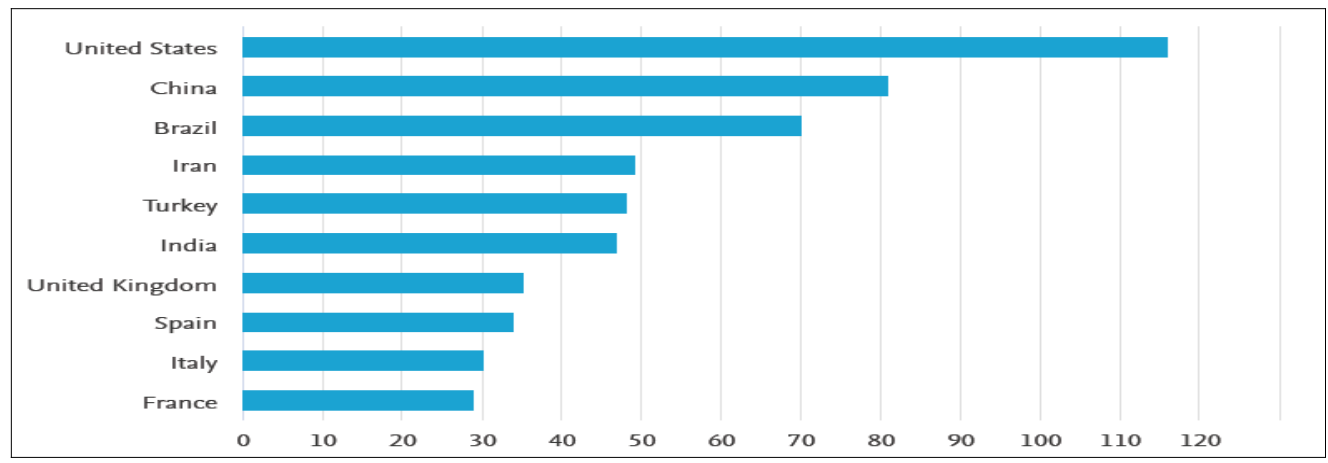

Gráfico 1: Análise bibliométrica. Fonte: Periódico Capes/Scopus - 1990 a 2018.

Conforme o Gráfico 2, verifica-se que o método pesquisado é de grande destaque, posto que ao longo dos anos de 1990 a 2018 houve um crescimento significativo nas publicações relacionadas ao tema.

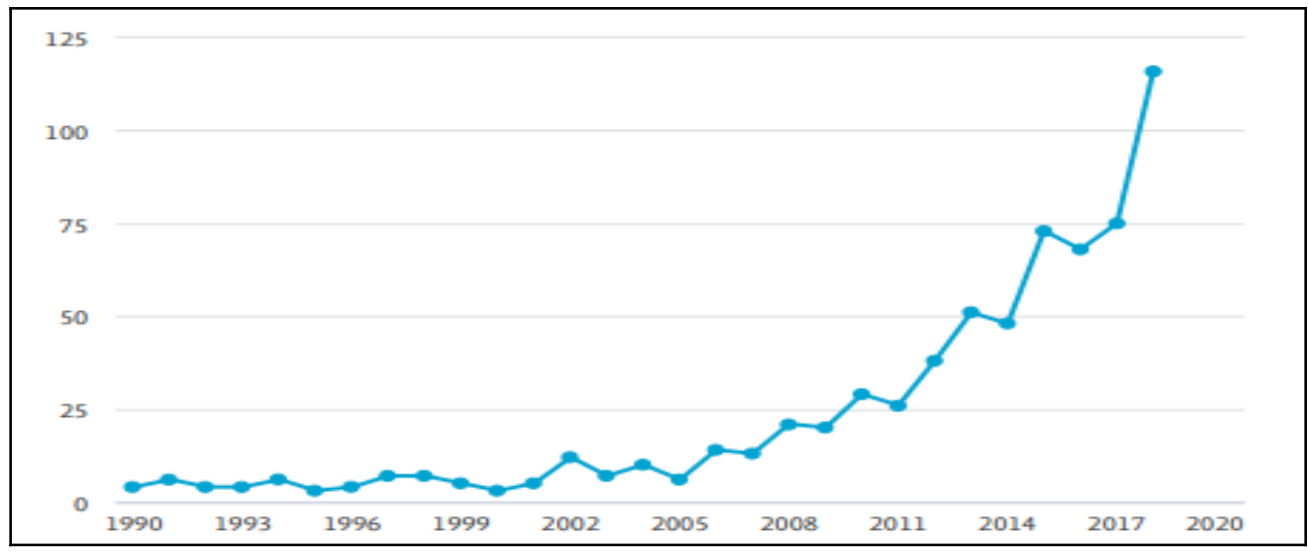

Gráfico 2: Análise bibliométrica. Fonte: Periódico Capes/Scopus - 1990 a 2018. 


\subsection{DIFERENTES MÉTODOS MULTICRITÉRIO}

Os métodos multicritério de tomada de decisão surgiram como métodos de apoio que são compreendidos como ferramentas matemáticas, eficazes para solução de problemas em que existem critérios conflitantes (Brans \& Mareschal, 2005).

A abordagem multicritério de apoio à decisão caracteriza-se como um grupo de métodos que buscam evidenciar um problema, no qual as alternativas são avaliadas por múltiplos critérios, sendo na maioria dos casos de caráter divergente (GOMES et al, 2002).

A seguir, dois dos métodos largamente utilizados para esses casos, AHP (Processo de Hierarquia Analítica) e (Elimination et Choix Traduisant la Réalite) ELECTRE, serão apresentados para compará-lo com o que será utilizado para o trabalho, o método Simple Aggregation of Preferences Expressed by Ordinal VectorsMulti Decision Makers (SAPEVO-M).

O Analytic Hierarchy Process - AHP foi um dos primeiros métodos de tomada de decisão com múltiplos critérios. Este surgiu durante a década de 1970 e foi desenvolvido pelo matemático Thomas L. Saaty.

O método tem como finalidade organizar os objetivos ou critério numa hierarquia representada pela preferência dos decisores. Além de o método AHP possibilitar a resolução de problemas com critérios conflitantes, uma das vantagens apontadas por Gomes (2009) se deve ao fato de o método permitir a participação de múltiplas pessoas, visto que é possível envolver diversos critérios e alternativas.

Saaty (1980) propôs o uso de uma escala padrão de valores, variando de 1 a 9, como forma de avaliar numericamente alternativas e critérios em um processo de decisão. Para cada valor, Saaty preocupou-se em definir seu predicado qualitativo seguido de explicação textual, para diminuir dúvidas no momento do julgamento.

O grupo de métodos ELECTRE são derivações do primeiro método apresentado por Bernard Roy em 1968. Teve início na escola francesa e possui característica de comparação par a par entre as alternativas, buscando uma relação de sobreclassificação entre elas, além de assumir a possibilidade de incomparabilidade na matriz de preferência do decisor.

Segundo Gomes, Araya e Carignano (2011), os métodos ELECTRE fazem parte dos denominados Métodos de Superação, posto que eles possuem como conceito teórico central, as relações de superação. Essas metodologias multicriteriais diferenciam-se entre si pela problemática que tentam resolver, pelas informações inter e intracritérios utilizadas e pela quantidade de relações de superação construídas e pesquisadas. A família ELECTRE é composta por seis tipos de versões (ELECTRE I, II, III, IV, IS e TRI).

Cada uma das versões ELECTRE difere operacionalmente e também se diferenciam em relação aos tipos de problemas que podem ser usados. Os métodos ELECTRE I, IV e IS são aplicáveis para uma problemática de escolha, onde o objetivo é selecionar um menor conjunto de melhores alternativas (ISHIZAKA; NEMERY, 2013; SIQUEIRA; ALMEIDA, 2011; OLIVEIRA et al., 2013). Já os métodos ELECTRE II, III e IV destinam-se à tarefa de construir uma ordem das alternativas do melhor para o pior.

Paralelamente, o ELECTRE II é baseado em critérios verdadeiros, enquanto que os outros dois métodos usam pseudo-critérios. Os métodos ELECTRE III e IV diferem em um número de pontos, mas a principal diferença é que o último não utiliza critérios pesos (GOVINDAN; JEPSEN, 2015). O ELECTRE TRI é um método multicritério de classificação, ou seja, um método que aloca alternativas em categorias pré-definidas (P.ß). A 
alocação de uma alternativa a resulta da comparação de a com perfis definidos de limites das categorias (MOUSSEAU \& SLOWINSKI, 1998).

\section{PROSPOSTA DE SOLUÇÃO}

\subsection{MODELAGEM MATEMÁTICA}

Existem diversos modelos de freezers disponíveis no mercado, porém para o desenvolvimento do trabalho foram selecionados três opções desses equipamentos que serão detalhados a seguir.

\section{Gelopar}

O modelo GCCP2P da Gelopar é de inox e composto de duas portas. O freezer possui controlador eletrônico digital com indicador de temperatura e degelo automático natural. $\mathrm{O}$ equipamento é capaz de alcançar uma temperatura que vai de $-20^{\circ} \mathrm{C}$ a $-12^{\circ} \mathrm{C}$, a refrigeração é de ar forçado com serpentina aletada, revestimento externo em aço inox 430 e interno aço galvanizado. Quanto às dimensões (CxAxL) 68x203x84,6 cm.

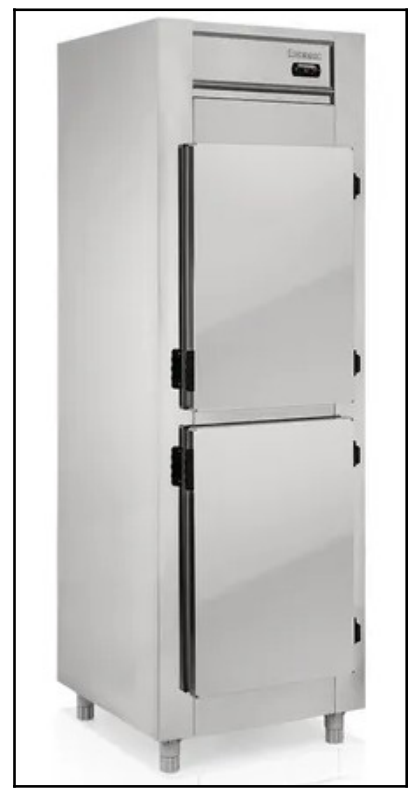

Figura 2: Modelo de freezer. Fonte: www.atau.com.br.

Características gerais:

- $\quad$ Marca: Gelopar

- $\quad$ Modelo: GCCP2P

- $\quad$ Capacidade: 536 litros

- $\quad$ Frente: $68 \mathrm{~cm}$

- $\quad$ Altura: 2,03 m

- $\quad$ Profundidade: $84 \mathrm{~cm}$

- Temperatura: $-22^{\circ} \mathrm{C}$ à $-12^{\circ} \mathrm{C}$

- $\quad$ Peso: $146 \mathrm{~kg}$

- $\quad$ Consumo: 11,4 kwh/dia

- $\quad$ Portas: 2 portas 


\section{Metalfrio}

O equipamento possui compressor de alta eficiência e condensador helicoidal, congela alimentos de forma rápida e eficiente, mesmo em condições climáticas extremas. Por meio de uma chave seletora, é possível escolher entre três funções: refrigerador de bebidas, conservador de gelos ou freezer para congelados. O modelo oferece uma faixa de temperatura específica para armazenar alimentos, seguindo os requisitos da Anvisa, dispensa a limpeza frequente e proporciona maior vida útil ao motor e ao compressor. É o equipamento de mais baixo consumo de energia da categoria.

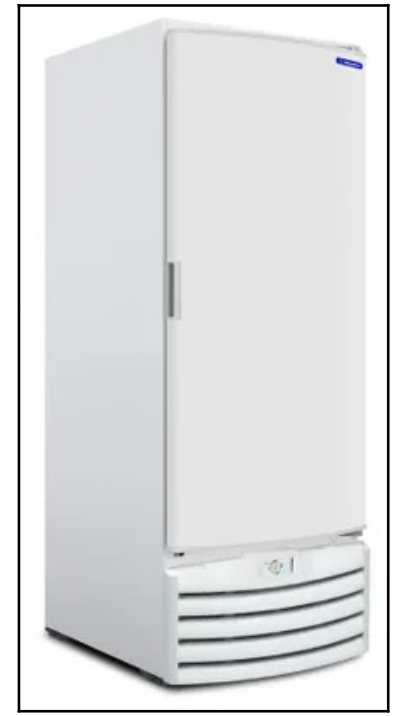

Figura 3: Modelo de freezer. Fonte: www.frigelar.com.br.

Características gerais:

- Marca: Metalfrio Tripla Ação

- Modelo: VF56

- Capacidade: 539 litros

- Largura: $675 \mathrm{~mm}$

- Altura: $1862 \mathrm{~mm}$

- Profundidade: $850 \mathrm{~mm}$

- Peso Líquido: 118

- Voltagem: $127 \mathrm{~V} / 220 \mathrm{~V}$

- Temperatura: freezer $-18^{\circ} \mathrm{C}$ à $-22^{\circ} \mathrm{C}$, conservador $-10^{\circ} \mathrm{C}$ à $-16^{\circ} \mathrm{C}$, refrigerador $2^{\circ}$ à $8^{\circ} \mathrm{C}$

\section{Frilux}

O freezer vertical modelo RF01 é utilizado para armazenagem e conservação de gelo, produtos previamente congelados e refrigerados em geral. Possui dupla ação, grade de ventilação plástica, aquecimento no quadro das portas, refrigeração estática e prateleiras reguláveis e inclináveis. Quanta à temperatura, a mesma varia entre $5^{\circ} \mathrm{C}$ à $-15^{\circ} \mathrm{C}$, possui capacidade de 560 litros e dimensões de (CxAxL) 66x200x76 cm. 


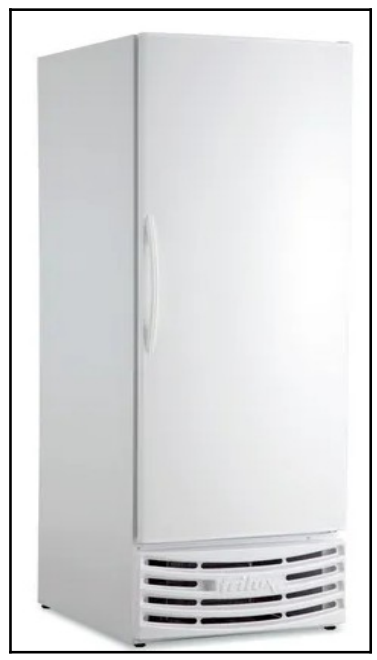

Figura 4: Modelo de freezer. Fonte:www.pollomaq.com.br.

Características gerais:

- Marca: Frilux

- Modelo: RF011

- Capacidade 560 litros

- Frente: $66 \mathrm{~cm}$

- Altura: 2,00 m

- Profundidade: $76 \mathrm{~cm}$

- Temperatura: $5{ }^{\circ} \mathrm{C}$ à $-15{ }^{\circ} \mathrm{C}$

- Peso: $102 \mathrm{~kg}$

- Consumo: 260 kwh/mês

- Portas: 1 porta

Com base nos modelos dos freezers apresentadas anteriormente, serão avaliados três critérios para a aplicação da ferramenta:

- CAPACIDADE: neste ponto será avaliada a medida em litros, ou seja, a quantidade de itens que cada freezer é capaz de acomodar.

- CUSTO DE AQUISIÇÃo: este critério é o valor em reais bruto para que seja possível efetuar a compra do equipamento.

- CUSTO DE MANUTENÇÃO: é o custo de todo e qualquer reparo que engloba mão de obra, ferramentas, peças de reposição, depreciação do equipamento, etc.

\subsection{DESENVOLVIMENTO DO MODELO MATEMÁTICO}

Para o desenvolvimento do trabalho foram realizadas entrevistas in loco semiestruturadas com os gerentes dos diferentes turnos do estabelecimento a fim de coletar as informações necessárias referente à problemática e verificar as condições reais do freezer avariado e, também dos outros existentes no estabelecimento. Para iniciar o desenvolvimento do modelo, é apresentada a Tabela 1 com a escala de critérios utilizada. 


\begin{tabular}{||l|l|l||}
\hline Escala 1 (símbolo) & Escala 1 (variável /expressão Linguística Correspondente) & Escala 2 \\
\hline$\ll \ll \ll 1$ & Absolutamente pior / Absolutamente menos importante & -3 \\
\hline$<\ll 1$ & Muito pior / Muito menos importante & -2 \\
\hline$\ll 1$ & Pior / Menos importante & -1 \\
\hline 1 & Igual ou equivalente / Tão importante quanto & 0 \\
\hline$» 1$ & Melhor / Mais importante & 1 \\
\hline$>» 1$ & Muito melhor / Muito mais importante & 2 \\
\hline$\gg \gg 1$ & Absolutamente melhor / Absolutamente mais importante & 3 \\
\hline
\end{tabular}

Tabela 1: Relação entre as opções de critérios/alternativas. Fonte: Gomes, Mury e Gomes (1997).

Após a identificação dos critérios a serem avaliados, os tomadores de decisão podem manifestar as suas opiniões a respeito dos critérios em ordem de preferência, conforme apresentado na Tabela 2, consequentemente gerando um "peso" para cada critério.

\begin{tabular}{|l|c|c|}
\hline \multicolumn{1}{|c|}{ Critérios } & Decisor 1: Renato & Decisor 2: Branca \\
\hline Capacidade VS Custo de aquisição & Melhor & Muito melhor \\
\hline Capacidade VS Custo de manutenção & Muito melhor & Melhor \\
\hline Custo de aquisição VS Custo de manutenção & Absolutamente melhor & Melhor \\
\hline
\end{tabular}

Tabela 2: Avaliação dos critérios pelos decisores. Fonte: Autores (2019).

Posteriormente, cada decisor pode estabelecer uma comparação paritária entre as alternativas dentro de cada critério, conforme apresentado na Tabela 3 e na Tabela 4.

\begin{tabular}{|l|c|c|c|}
\hline \multicolumn{1}{|c|}{ Decisor 1: Renato } & Gelopar VS Metalfrio & Gelopar VS Frilux & Metalfrio VS Frilux \\
\hline Critério Capacidade & Equivalente & Muito pior & Pior \\
\hline Critério Custo de aquisição & Absolutamente pior & Muito pior & Pior \\
\hline Critério Custo de manutenção & Muito pior & Pior & Equivalente \\
\hline
\end{tabular}

Tabela 3: Avaliação dos critérios pelo decisor 1 em relação aos freezers. Fonte: Autores (2019).

\begin{tabular}{|l|c|c|c||}
\hline \multicolumn{1}{|c|}{ Decisor 2: Branca } & Gelopar VS Metalfio & Gelopar VS Frilux & Metalfrio VS Frilux \\
\hline Critério Capacidade & Pior & Muito pior & Muito pior \\
\hline Critério Custo de aquisicção & Absolutamente pior & Absolutamente pior & Equivalente \\
\hline Critério Custo de manutenção & Pior & Muito pior & Melhor \\
\hline \hline
\end{tabular}

Tabela 4: Avaliação dos critérios pelo decisor 2 em relação aos freezers. Fonte: Autores (2019). 


\section{RESULTADOS ALCANÇADOS}

Para o cálculo dos resultados do método SAPEVO-M, utilizou-se o aplicativo computacional SAPEVO Web, desenvolvido pelo Instituto Militar de Engenharia (IME) em parceria com a Universidade Federal Fluminense (UFF), podendo ser acessado pelo site www.sapevoweb.com.

Em concordância com o desenvolvimento do modelo matemático, baseado nas opiniões dos tomadores de decisão, observou-se que o freezer melhor classificado foi o da marca Frilux, ficando em primeiro lugar, seguido do modelo Metalfrio e na última colocação o freezer da marca Gelopar.

Nesse sentido, é possível notar que as cardinalidades finais geradas para cada alternativa de freezer foram muito díspares, na qual o último colocado ficou sem possibilidade alguma de ser escolhido como opção de compra.

Vale ressaltar que outro aspecto relevante acerca da melhor escolha encontrada é de que o modelo Frilux possui a maior capacidade de armazenamento de pães entre as demais opções. Dessa forma, pode-se dizer que será possível também o aumento da produção de pães na padaria e em consequência disso o melhor atendimento aos clientes.

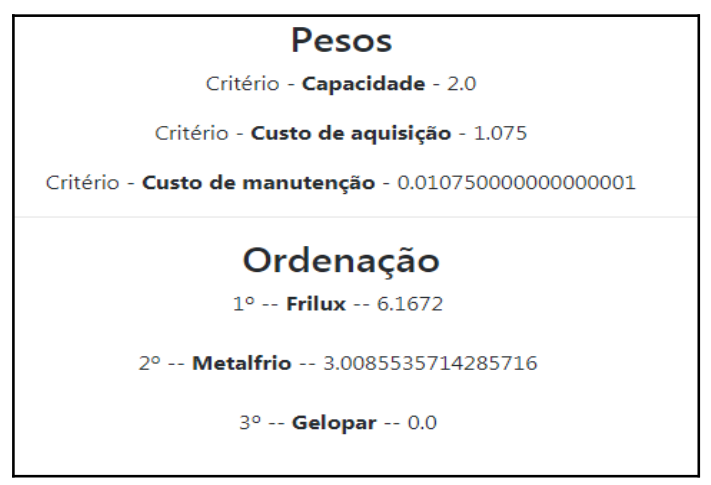

Figura 5: Resultado final. Fonte: Autores (2019)

\section{CONSIDERAÇÕES FINAIS}

Conforme foi observado no presente trabalho, o método SAPEVO-M, aplicado em diferentes situações, mostrou-se adequado na modelagem do problema abordado no estudo de caso.

Primeiramente, foi identificado o problema e constatou-se a necessidade de adquirir um novo freezer que fosse capaz de atender à demanda da padaria. Por meio do método SAPEVO-M, foi possível obter a classificação final do freezer que teve melhor resultado dentre os três modelos apresentados no presente estudo.

Por intermédio do resultado esperado, o objetivo desta ferramenta é auxiliar na tomada de decisão de uma forma clara e objetiva buscando assim contribuir na resolução de um determinado problema. Levando em consideração a ferramenta estudada é possível notar sua gama de aplicabilidade. Pode ser utilizada para resolução de problemas dos mais variados tipos, sendo assim um método de grande utilidade voltado para a contribuição da tomada de decisão.

Dessa forma, com a aplicação do método SAPEVO-M, conseguiu-se propor uma metodologia de auxílio na tomada de decisão, para que os decisores tenham um maior 
embasamento técnico, analítico e quantitativo na seleção do freezer a ser adquirido. Os freezers avaliados foram ordenados de maneira clara e coerente segundo os critérios estabelecidos pelos próprios decisores.

\section{REERÊNCIAS BIBLIOGRÁFICAS}

AZEVEDO, I. B. Se meu povo orar. 1. Ed. Rio de Janeiro: MK Editora, 2003. 157p.

BRANS, J. P.; MARESCHAL, B. How to Decide with PROMETHEE, 1990. Disponível em: http://www.visualdecision.com/dlab methods.htm.

FERREIRA, P. H. G.; AMORIM, R. M.; MACIEL, R.H. Panificação e Gestão estratégica de Custos: o alcance da vantagem competitiva no setor, 2004.

GOMES, K. G. A. (2009). Um método multicritério para localização de unidades de celulares de intendência da FAB (Dissertação de mestrado). Departamento de Engenharia de Produção, Pontifícia Universidade Católica do Rio de Janeiro, Rio de Janeiro.

GOMES, L. F. A. M.; ARAYA, M. C. G.; CARIGNANO, C. Tomada de decisões em cenários complexos. São Paulo: Cengage Learning, 2011.

GOMES, L. F. A. M., MURY, A. R., GOMES, C. F. S. Multicriteria Ranking with Ordinal Data. SAMS, vol. 27, pp. $139-145$.

GOVINDAN, Kannan; JEPSEN, Martin Brandt. ELECTRE: A comprehensive literature review on methodologies and applications. European Journal of Operational Research, [s.l.], v. 250, n. 1, p.1-29, abr. 2016.

ISHIZAKA, A; NEMERY, P. Multi-Criteria Decision Analysis: Methods and Softwares. Wiley, 2013.

SAATY, Thomas L. Decision Making for Leaders - The analytic Hierarchy Process for Decisions in a Complex World. Pittsburg: University of Pittsburg, 1995.

SERVIÇO BRASILEIRO DE APOIO ÀS MICRO E PEQUENAS EMPRESAS - SEBRAE. Pães Caseiros Não Industrializados. Estudos de Mercado. SEBRAE/ESPM. Relatório Completo. Março de 2008. 149p. (2008). Disponível em http://bis.sebrae.com.br/GestorRepositorio/ARQUIVOS_CHRONUS/bds/bds.nsf/0E7 3F943EC870F468325753E005F5A80/\$File/NT0003DB1A.pdf.

TEIXEIRA, P. C. et al. Padronização e melhoria de processos produtivos em empresas de panificação: estudo de múltiplos casos. Production, v. 24, n. 2, p. 311-321, 2014. 